\title{
Human capital and growth cycles
}

\begin{abstract}
This paper studies the dynamic interaction between human capital accumulation and economic growth. Capital market imperfections and an indivisibility in human capital investment prevent poor agents from accumulating skills, the acquisition of which positively affects technological progress. More productive technologies in turn require more sophisticated qualificatio and involve higher training costs. The equilibrium dynamics can be characterized by the joint evolution of productivity growth, the schooling costs, and the income distribution. Under our assumptions, individual incomes follow a non-linear Markov chain. This nonlinearity generates endogenous fluctuation of schooling activities and the rate at which productivity improvements occur.
\end{abstract}

Keywords Human capital accumulation - Income distribution - Capital market imperfection · Endogenous growth · Cycles

JEL Classificatio Numbers: D31 $\cdot$ E32 $\cdot$ O11 $\cdot$ O16

\section{Introduction}

In industrialized countries, average per-capita output grows at a rate that is bounded away from zero. At the same time, economic activity undergoes a cyclical

We thank an anonymous referee for many helpful suggestions. Support from the German Research Foundation (DFG) under grant KA1519/2-2 is gratefully acknowledged.

L. Kaas

Department of Economics, University of Konstanz, 78457 Konstanz, Germany

E-mail: leo.kaas@uni-konstanz.de

S. Zink ( $\varangle)$

Department of Economics, University of Konstanz, 78457 Konstanz, Germany

E-mail: stefan.zink@uni-konstanz.de 
development. Traditionally, economists try to explain these observations separately, with growth theorists analyzing the trend path of economies and business cycle researchers focusing on detrended data. Moreover, while trend growth is seen to reflec technological and institutional characteristics of economies, the dominating view concerning fluctuation is that they are a response to exogenous shocks. In the present paper, we argue that productivity growth quite naturally has a cyclical component which gives rise to fluctuations i.e. even in the absence of any shocks, economies cycle back and forth between periods of high and low productivity growth. Thus, growth and cycles are endogenous and related phenomena.

Crucial for our mechanism is the interplay between human capital accumulation and productivity growth. Educated persons not only acquire productive skills, but they learn how to learn (see Nelson and Phelps 1966) which enables them to deal with a changing environment. Being able to understand technology and the production process, moreover, makes them good innovators. There is therefore some agreement that average human capital in a society has a positive effect on productivity growth and the implementation of new technologies. ${ }^{1}$ The reverse causality is, however, also likely to be of relevance. Complex technologies can only be operated by workers who have acquired certain technology-specifi skills. As machines become more productive, qualificatio requirements typically change. If schooling is costly and if innovations are skill biased in the sense that "new skills are more costly to acquire than skills required by preexisting types of equipment" (Caselli 1999, p.78), technological improvements may have an impact on people's incentives and ability to invest in the corresponding qualification In the present paper, we take account of both causalities - from human capital accumulation to productivity growth and from productivity growth to human capital accumulation. In particular, we show that the two-way interaction generates endogenous fluc tuations of schooling activities and the rate at which technology improvements occur. $^{2}$

In our model, capital market imperfections and a non-convexity in human capital investment prevent poor agents from accumulating skills, the acquisition of which positively affects technological progress. Moreover, technological improvements raise the costs of education and training. The equilibrium dynamics can be characterized by the joint evolution of the growth rate, the education costs, and the income distribution. Since the development of technology and the education costs depends on the aggregate amount of human capital in the economy, the path of a single household's income is influence by the schooling decisions of other families. These external effects of human capital accumulation create a non-linearity which is ultimately responsible for a cyclical development of productivity growth and skill acquisition. By contrast, other authors also investigating models with non-linear transition functions (cf., for instance, Owen and Weil 1998, or Piketty 1997) focus on the multiplicity of equilibria following from the non-linearity.

Our work is associated with a strand of literature which studies reasons why growth quite naturally follows a cyclical pattern. Related articles include, for instance, Wälde (2002), Boldrin and Levine (2001), Matsuyama (2001, 1999), or

\footnotetext{
${ }^{1}$ Evidence supporting this view is discussed in Schultz (1975) as well as Bartel and Lichtenberg (1987).

2 Dellas and Sakellaris (2003) as well as DeJong and Ingram (2001) document and analyze the observation that skill acquisition is subject to significan f uctuations.
} 
Evans et al. (1998). While these works build on the interplay of gradual improvements of existing production possibilities and major technological innovations (Boldrin and Levine 2001), on a switching between a regime of innovation and a regime of capital accumulation (Wälde 2002; Matsuyama 2001, 1999), or on sunspots and expectational indeterminacy (Evans et al. 1998) to explain growth in cycles, we highlight the intertemporal linkage between human capital accumulation and productivity growth.

Our paper, moreover, relates to research on the nexus between inequality and growth under imperfect capital markets. The early literature is reviewed in Benabou (1996). More recent articles include, for instance, De Gregorio and Kim (2000) or Chiu (1998). While most contributions concentrate either on the influenc of inequality on growth or on the reverse causality, we study both directions in a unifie framework.

The rest of the paper is organized as follows. Section 2 describes our basic model structure and the individuals' investment problem. We analyze the resulting dynamics in Section 3 and discuss an extension in Section 4. The paper closes with some concluding remarks.

\section{The environment}

\subsection{Individuals}

Our economy consists of overlapping generations of individuals and a single nonstorable good. Each generation comprises a continuum of individuals of mass 1 . Every single person lives for two periods and has one parent and one child. In the firs period of life, individuals can go to school and acquire skills. In the second period, they work, as either skilled or unskilled, consume, and invest in the education of their child. Individuals care about their own consumption $c_{t}$ and about the income which their child earns during working life $y_{t+1}$. Their preferences are represented by a utility function $u\left(c_{t}, y_{t+1}\right)$ which is assumed to be differentiable, homothetic, strictly increasing, and strictly quasi-concave. Because our model has income growth, homotheticity is assumed to make sure that education decisions are independent of the current level of aggregate income. ${ }^{3}$

\subsection{Technology}

Production in period $t$ occurs with a technology that can be described by a scalar index $V_{t}$ measuring aggregate productivity. Production is linear in labor so that aggregate output in the economy equals $V_{t} H_{t}$ where $H_{t}$ denotes the total number of efficien labor units. A low skilled person supplies one unit of efficien labor, thus earning $V_{t}$. Acquiring certain technology-specifi skills raises the number of efficien labor units to $m>1$. Let $n_{t}$ denote the number of persons working as high skilled in period $t$, so that $H_{t}=n_{t} m+\left(1-n_{t}\right)$.

Empirical evidence shows that human capital is conducive for technological progress. To formalize this link we assume that aggregate productivity $V$ grows

\footnotetext{
${ }^{3}$ In Section 4 we show that our results are not confine to this particular preference structure.
} 
at a rate which is positively affected by the number of educated people, $n$, in the production process. ${ }^{4}$ We capture the relationship between the size of the educated work force and productivity growth by a function $\Lambda\left(n_{t}\right)$ which attains only two values. The low value $\Lambda$ is adopted if the number of educated persons is below some threshold value $\tilde{n} \in(0,1)$. If the number of high-skilled persons exceeds $\tilde{n}$, productivity grows at the larger rate $\bar{\Lambda}$. Therefore productivity growth takes the following form: 5

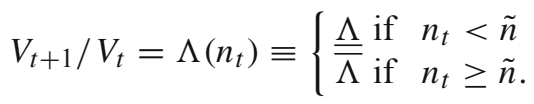

It is crucial for the model that production in $t$ has an influenc on the state of technology available in $t+1$, leaving labor productivity in $t$ unaffected. This might formalize the idea that it takes some time until increased experience or knowledge translate into efficien y gains.

\subsection{Education decisions}

While working as low skilled involves no costs, being high skilled requires an investment by the parents. We assume that the education costs depend positively on the productivity level of the technology that the individual wishes to apply. This captures the idea that more sophisticated equipment requires more sophisticated qualification On the other hand, the individual costs of education are also affected by innate ability. The extent of inaptitude, which is inversely related to cognitive ability, is measured by some variable $h$. We assume that $h$ is a random variable which is independent across families and over time. The stochastic nature of innate ability allows for intergenerational social mobility which we observe in the data (cf., for instance, Erikson and Goldthorpe 1992). The distribution of $h$ is uniform over the interval $[0,1]$. In period $t$, parents learn the innate ability $h$ of their child and they also correctly anticipate next period's technology level $V_{t+1}$ that their child will be working with. Given $h$ and $V_{t+1}$, the costs of skill acquisition in period $t$ are $h V_{t+1} .^{6}$

The availability of educational loans is limited since they cannot be backed by human capital. In particular, parents cannot commit their children to repay their educational loan. We consequently assume that, when investing in their children's education, parents have to rely on their income without access to external funds.

\footnotetext{
${ }^{4}$ Note that $H$ - the total number of eff cient labor units - is strictly increasing in $n$. Hence, using other measures of human capital - like, for instance, average human capital - would not change our qualitative results.

5 Technologies with threshold properties have been used in the growth literature to deliver multiple equilibria and development traps (see e.g. Azariadis and Drazen 1990; Azariadis 1996). To our knowledge, however, such models do not readily give rise to endogenous growth cycles as our model. We show below that cycles also emerge for other classes of technologies exhibiting a positive link between human capital and technological progress.

6 The sequence of events within every period can be summarized as follows: (1) production occurs with productivity $V_{t},(2)$ due to spillover effects from the production process, a better technology becomes available: $V_{t+1}=V_{t} \Lambda\left(n_{t}\right)$, (3) parents realize their children's cognitive ability $h$, (4) parents decide over consumption and their offspring's educational attainment taking $V_{t+1}$ and $h$ as given.
} 
Due to the indivisibility in investment, parents compare their utilities with and without investing in their children's education.

Consider a parent in period $t$ with income $y_{t}=m_{t} V_{t}$ where $m_{t}=1$ if the individual is unskilled, and $m_{t}=m$ if the individual is skilled. The parent decides to educate his child if, and only if,

$$
u\left(y_{t}-h V_{t+1}, m V_{t+1}\right) \geq u\left(y_{t}, V_{t+1}\right) .
$$

Using homotheticity and (1) this condition is the same as

$$
u\left(\frac{m_{t}}{\Lambda\left(n_{t}\right)}-h, m\right) \geq u\left(\frac{m_{t}}{\Lambda\left(n_{t}\right)}, 1\right) .
$$

This shows that there is a threshold level $\tilde{h}=H\left(\frac{m_{t}}{\Lambda\left(n_{t}\right)}\right)$ which satisfie (2) with equality such that parents whose child has inaptitude $h \leq \tilde{h}$ educate their children, and parents whose child has inaptitude $h>\tilde{h}$ do not educate their children. ${ }^{7}$ In the Appendix we show the following:

Lemma 1 The threshold function $H(x)$ defined by $u(x-H(x), m)=u(x, 1)$ is strictly increasing in $x$.

The lemma implies that parents are more likely to educate their children if they are skilled themselves: skilled (and richer) households suffer from a smaller utility sacrific when educating their children and are consequently more likely to do so. Another conclusion of this lemma is that parents are less likely to educate their children when productivity growth is fast: if productivity increases at a high rate (i.e. $\Lambda\left(n_{t}\right)$ is large), schooling costs are large which makes it less attractive to invest in education.

Because $m_{t} / \Lambda\left(n_{t}\right)$ attains only four values when $\Lambda\left(n_{t}\right)$ has the form (1), we only need to focus on four different threshold values. We assume that the largest of them (the one for skilled parents under low productivity growth) is strictly smaller than one. This implies that all parents, irrespective of their income and of productivity growth, decide not to educate children with very low ability. Specificall we assume

$$
H\left(\frac{m}{\underline{\Lambda}}\right)<1 \quad \text { or } \quad u\left(\frac{m}{\underline{\Lambda}}-1, m\right)<u\left(\frac{m}{\Lambda}, 1\right) .
$$

\section{Dynamics}

Let us suppose firs that aggregate productivity grows at a constant exogenous rate, so that $\Lambda=\bar{\Lambda}=\Lambda$. In this case, the evolution of individual families can be studied in isolation from other households. There are fi ed threshold abilities $\widetilde{h}^{S}=H(m / \Lambda)$ for skilled and $\widetilde{h}^{U}=H(1 / \Lambda)$ for unskilled workers. Given a certain profession in one generation, the household maintains this occupation with a constant probability and switches to the other profession with the counter probability. Regardless of the initial distribution of occupations, this Markov structure implies convergence

\footnotetext{
${ }^{7}$ To give an example, when the utility function has the Cobb-Douglas form $\ln c+\beta \ln y$, the threshold function is $H(x)=\left(1-m^{-\beta}\right) x$.
} 
to an ergodic distribution in the long run. ${ }^{8}$ Higher productivity growth makes it more expensive in terms of forgone utility to invest in education and therefore lowers both $\widetilde{h}^{S}$ and $\widetilde{h}^{U}$. This in turn leads to fewer skilled workers in the long run. The intuition for this result is the following. People's income in $t$ depends on the technology in that period, whereas the education costs depend on the technology in $t+1$. A large value of $\Lambda$ increases the difference in the state of technology between generations. It is therefore easier to financ human capital accumulation if productivity grows at a lower rate.

If $\Lambda$ is endogenous, it is no longer possible to study the dynamics of single households isolated from the rest of the population because society's educational attainment is crucial for the development of $V$ and the skill requirements. Individual (detrended) income follows a non-linear Markov chain which, as we will see, may generate endogenous fluctuation of schooling activities and the rate at which productivity improvements occur. ${ }^{9}$ In our model, the evolution of the (detrended) income distribution boils down to the dynamics of the single state variable $n_{t}$, which allows us to characterize the dynamic path analytically. This result follows from two simplifying assumptions: (1) there are only two professions and the skill premium is exogenously given; (2) parents bequeath professions to their children instead of monetary transfers. Without these simplifications we would have to deal with an infinite-dimensiona state variable.

We can describe how the share of skilled workers in the population, $n_{t}$, changes over time. Because of (A1) and since we have a continuum of households and a uniform distribution of $h$, the evolution of $n_{t}$ satisfie the following first-orde difference equation:

$$
n_{t+1}=\Phi\left(n_{t}\right) \equiv H\left(\frac{m}{\Lambda\left(n_{t}\right)}\right) n_{t}+H\left(\frac{1}{\Lambda\left(n_{t}\right)}\right)\left(1-n_{t}\right) .
$$

Note that assumption (A1) and Lemma 1 imply that $\Phi$ maps the unit interval $[0,1]$ into itself. Equation (3) shows that the occupational structure in one generation, as represented by $n_{t}$, affects the number of skilled persons in the subsequent generation in two different ways. On the one hand, there is a link from distribution to growth through $\Lambda$. On the other hand, richer families fin it easier to buy education in the absence of education loans. A larger number of skilled households therefore translates into more skilled children.

Given the threshold property of $\Lambda\left(n_{t}\right)$, the function $\Phi$ takes the following form:

$$
n_{t+1}=\Phi\left(n_{t}\right)= \begin{cases}\Phi_{1}\left(n_{t}\right) \equiv a_{1}+b_{1} n_{t} & \text { if } n_{t}<\tilde{n} \\ \Phi_{2}\left(n_{t}\right) \equiv a_{2}+b_{2} n_{t} & \text { if } \quad n_{t} \geq \tilde{n}\end{cases}
$$

\footnotetext{
${ }^{8}$ In our model, a non-degenerate qualificatio structure arises because agents receive independent ability shocks and come from different family background, which both affects the willingness and ability to invest in education. Be careful to note that the distribution of professions is equivalent to the distribution of detrended incomes $y_{t} / V_{t}$.

9 Other examples of non-linear Markov chains in the economic literature include, for instance, Owen and Weil (1998) or Piketty (1997). In their work, the equilibrium wage (Owen and Weil 1998) or interest rate (Piketty 1997) depends on the current wealth distribution because there are capital market imperfections. Hence, the evolution of lineage wealth is affected by the development of overall inequality. Due to this non-linearity, multiple equilibrium wealth distributions can emerge in their models.
} 


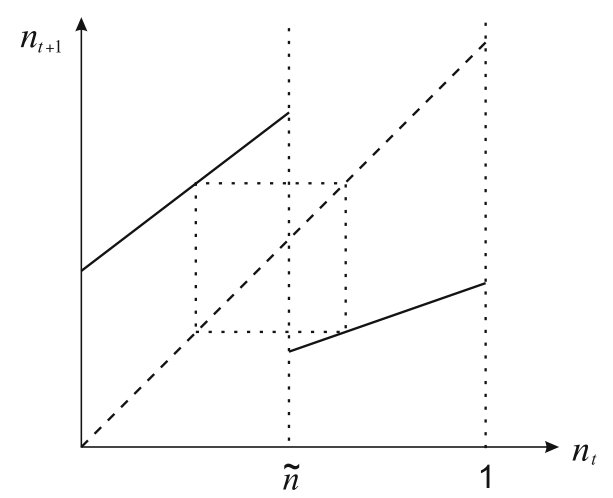

Fig. 1 A cycle of period 2

The parameters $a_{i}$ and $b_{i}, i=1,2$ are all positive, strictly smaller than one and are define by

$$
\begin{gathered}
a_{1}=H\left(\frac{1}{\underline{\Lambda}}\right)>a_{2}=H\left(\frac{1}{\bar{\Lambda}}\right), \\
b_{1}=H\left(\frac{m}{\underline{M}}\right)-a_{1}, b_{2}=H\left(\frac{m}{\bar{\Lambda}}\right)-a_{2} .
\end{gathered}
$$

Because of Lemma 1, $\Phi$ jumps down at the threshold level $\tilde{n}$. Therefore, there can exist at most one steady state $n^{*}$ which must be globally stable. However, for some parameters, the dynamics will not settle down to a stationary equilibrium. Particularly, if

$$
\Phi_{1}(\tilde{n})>\tilde{n}>\Phi_{2}(\tilde{n})
$$

holds, there does not exist a steady state, and the economy must fluctuat permanently between high- and low-growth regimes. Generally, one can fin cycles of any order by choosing the underlying parameters appropriately. To give an example, the necessary and sufficien condition for the existence of a cycle of period 2 is $a_{2}+b_{2} a_{1}<\left(1-b_{1} b_{2}\right) \tilde{n} \leq a_{1}+b_{1} a_{2}$. Figure 1 illustrates such a situation.

For other parameter configurations cycles which spend long phases in regimes of high or low productivity growth also emerge, as we illustrate below. It is a consequence of the particular piecewise linear structure of this model, that any cycle is globally stable. ${ }^{10}$ In particular, for any given parameter combination, there exists at most one such cycle and all trajectories converge to it, irrespective of the initial condition.

Proposition 1 When (5) holds, the economy fluctuates between regimes of high and low growth. If there exists a cycle of period $\tau>1$, it is globally stable.

Proof Appendix.

${ }^{10}$ A similar result for such a piecewise linear dynamic system can be found in Böhm and Kaas (2000). In fact one can show that such periodic cycles exist except for parameter values in a set of measure zero. 
What is the intuition behind the growth cycles in our model? If the number of skilled persons is large, productivity increases at a high rate. Large productivity improvements change the requirements of becoming skilled, thus driving up the training costs. This makes investing in education less attractive and lowers the cost threshold values. A lower share of skilled workers in turn slows down technological progress. When growth rates are moderate, the production technology and the skills necessary to apply it do not change very rapidly. This makes it cheaper to invest in skills. A larger stock of educated workers in turn speeds up the implementation of innovative technologies. As a result, the economy cycles between periods of high and low growth.

What behavior an economy experiences in the long run - low or high steady growth, or cycles - depends critically on the utility function and the underlying parameters. Generally, it can be said that configuration where incentives to invest in human capital are particularly strong or weak lead to stable situations, while the intermediate situation is conducive for volatility. To illustrate this assertion, we study how the dynamic behavior depends on the level of the skill premium $m$. Technically, both functions $\Phi_{1}$ and $\Phi_{2}$ shift upward as $m$ increases. ${ }^{11}$ Figure 2 illustrates what dynamic behavior our model predicts when the skill premium is increased. In Fig. 2a the skill premium is so low that the unique stable steady state is in the regime of low growth. Not enough parents invest in education for growth to take off. When the skill premium is increased, Fig. $2 \mathrm{~b}$ and $\mathrm{c}$ shows that the steady state ceases to exist; the asymptotic behavior follows a cyclical pattern between regimes of high and low growth. In Fig. $2 b$ the skill premium is smaller than in Fig. 2c: consequently, cycles in Fig. 2b spend longer phases in the regime of low growth than the cycles in Fig. 2c. Finally, when the skill premium is increased even further, a stable steady state in the regime of high growth emerges and volatility disappears.

In the above analysis, cycles occur because of a discontinuity which prevents the existence of a steady state. One might object that the existence of a human capital threshold is a rather special assumption. However, economies may also exhibit cyclical behavior if productivity growth advances smoothly with the share of skilled workers $n$. Suppose that a strictly increasing function $\Lambda \in C^{1}$ maps the interval $[0,1]$ onto $[\Lambda, \bar{\Lambda}] \subset[1,+\infty)$. Under (A1), $\Phi(0)>0$ and $\Phi(1)<1$ guarantee the existence of a fi ed point of (3). Any steady state satisfie

$$
1=H\left(\frac{m}{\Lambda\left(n^{*}\right)}\right)+H\left(\frac{1}{\Lambda\left(n^{*}\right)}\right)\left(\frac{1}{n^{*}}-1\right)
$$

and must be unique because of Lemma 1 . The shape of the graph of $\Phi$ is not generally clear under smooth technological progress. Increasing $n_{t}$ can lead to more or fewer high skilled workers. On the one hand, higher $n_{t}$ implies that there are more families who achieve the higher threshold value $\widetilde{h}^{S}$. On the other hand, a larger skilled labor force implies higher productivity growth which makes it more expensive to invest in the more productive technology and thus discourages skill acquisition. If the steady state lies within a downward-sloping segment of the phase

11 A higher skill premium raises all agents' incentives to invest. Moreover, the ability of skilled households to financ education improves. 


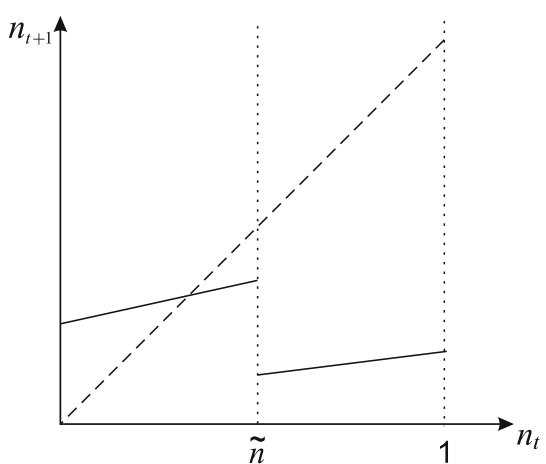

(a) Low growth without fluctuations

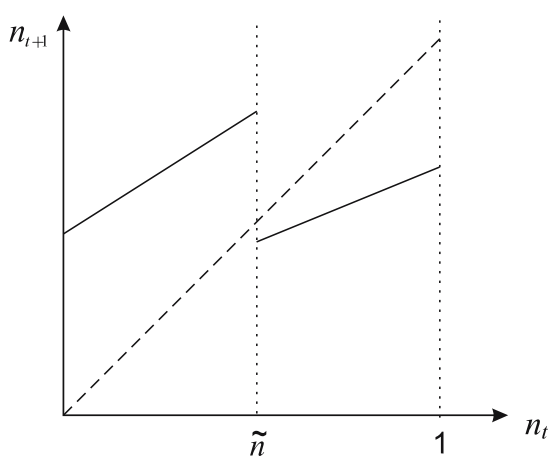

(c) High growth with fluctuations

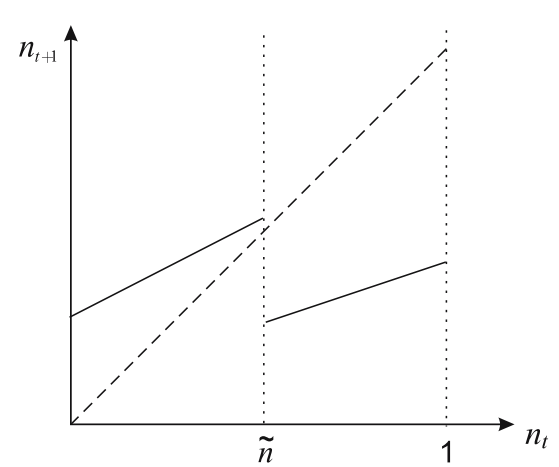

(b) Low growth with fluctuations

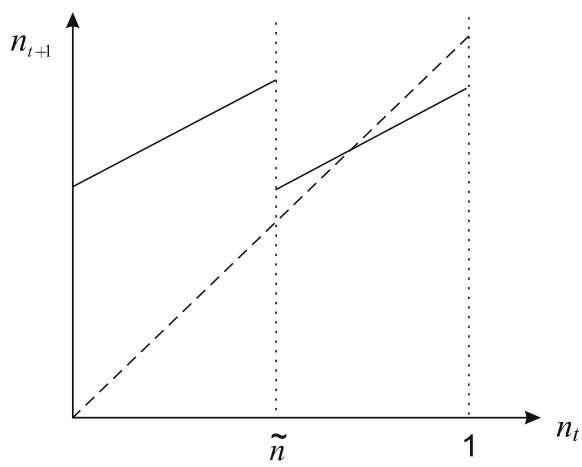

(d) High growth without fluctuations

Fig. 2 a-d Different dynamic patterns as the skill premium increases

line, the dynamics of $n_{t}$ will display non-monotonicities. ${ }^{12}$ Any smooth approximation of the step function (1) is, for example, sufficien for a cyclical development pattern. ${ }^{13}$

\section{Dynastic preferences}

This section shows that the qualitative results do not hinge on the above preference structure. In particular, we show that growth cycles may also obtain under a dynastic utility function. We assume that each generation maximizes an additive function of their one-period utility $\ln c_{t}$ and the utility of the subsequent generation $U_{t+1}$ discounted by $\beta \in(0,1)$. Individuals maximize their utility, taking the evolution of the aggregate state variable $V_{t}$, and thus productivity growth as given.

\footnotetext{
12 To give an example, when the utility function is $\ln (c)+\beta \ln (y)$ and $\Lambda(n)=1+b n, b>m-1$ is suff cient for cyclical convergence to a globally stable steady state.

13 The dynamics of this model can be expected to exhibit quite irregular patterns. Paralleling the proof of Proposition 5 in Böhm and Kaas (2000), we can show that period-3 cycles exist which are suff cient for topological chaos in the sense of Li and Yorke.
} 
Apart from the preferences, the basic model structure remains unaltered. We focus on technological progress with the threshold property (1), and the investment decision is as before: knowing $h$ the parent chooses whether to purchase education for the child or not: $\delta_{t}=1$ or $\delta_{t}=0$. In case of education, the child works as skilled, otherwise as unskilled. ${ }^{14}$ The maximization problem can then be written as

$$
\max E\left\{\sum_{t=0}^{\infty} \beta^{t} \ln \left(y_{t}-\delta_{t} h_{t} V_{t+1}\right)\right\}
$$

subject to the constraints $y_{t}=\left[1+\delta_{t-1}(m-1)\right] V_{t}$. The individual takes $\left(V_{t}\right)_{t=0}^{\infty}$ and $y_{0}$ as given. The expectation is over the sequence of future education costs $\left(h_{t}\right)_{t=1}^{\infty}$. To transform the model into a stationary form, we look at detrended variables: $m_{t}=y_{t} / V_{t}=\delta_{t-1} m+\left(1-\delta_{t-1}\right)$ and $h_{t} V_{t+1} / V_{t}=\Lambda_{t} h_{t}$.

Using this normalization the objective function is

$$
\sum_{t=0}^{\infty} \beta^{t}\left[\ln \left(m_{t}-\delta_{t} \Lambda_{t} h_{t}\right)+\ln V_{t}\right] .
$$

The fact that $V_{t}$ is given for the individual leaves us with the following maximization problem:

$$
\max E\left\{\sum_{t=0}^{\infty} \beta^{t} \ln \left(m_{t}-\delta_{t} \Lambda_{t} h_{t}\right)\right\} .
$$

subject to the constraints $m_{t}=1+\delta_{t-1}(m-1)$, where $m_{0}$ and the sequence of technological progress $\left(\Lambda_{t}\right)_{t=0}^{\infty}$ are given. As before, avoiding corner solutions for $\widetilde{h}^{S}$ requires a condition like (A1). We impose $m<\underline{\Lambda}$ so that the least talented of the rich are not able to financ education.

Up to this point, we have focused on one family which chooses its occupation taking aggregate variables as given. For given $n_{0}$ and thus $\Lambda_{0}$, an equilibrium in the whole economy is a sequence $\left\{n_{t}, \Lambda_{t}\right\}_{t=0}^{\infty}$ such that

- the investment strategies following from the maximization problem (6) imply the sequence $\left(n_{t}\right)_{t=0}^{\infty}$.

- in each period

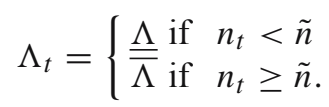

A pair $\left(n^{*}, \Lambda^{*}\right)$ is called a steady state if there exists an equilibrium with $n_{t}=n^{*}$ for all $t$. It is generally difficul to describe the distributional dynamics in the endogenous growth case. However, we can derive a condition similar to (5) under which no equilibrium sequence $\left(\Lambda_{t}\right)_{t=0}^{\infty}$ converges, i.e. neither $n_{t}<\tilde{n}$ nor $n_{t} \geq \tilde{n}$ can

14 One may also interpret this framework as a single-agent model with an infinit planning horizon and idiosyncratic ability shocks. Then, after each period the technology-specifi skills either become obsolete or depreciate fully. To incorporate a comparative advantage in 'learning' the new technology for the previously skilled, one might still assume that they have lower education costs $h V_{t+1} / k$ where $k>1$, but this would not change the results. 
hold infinitel often in succession. For this purpose, assume there is an equilibrium sequence such that $\Lambda_{t}$ does no longer change from period $k$ onward, so that $\Lambda_{k+t}=\Lambda \in\{\underline{\Lambda}, \bar{\Lambda}\}$ for all $t$. Because the utility maximization problem (6) only depends on $\Lambda_{t}$ but not on $n_{t}$, this problem is stationary from period $k$ onward. Starting with period $k$, the optimal stationary investment strategy is described as follows:

Proposition 2 For a given constant productivity growth factor $\Lambda$, skilled (unskilled) households invest in education if the realization of $h$ lies below some threshold value $\widetilde{h}^{S}(\Lambda)\left(\widetilde{h}^{U}(\Lambda)\right)$ where $\widetilde{h}^{S}(\Lambda)=m \widetilde{h}^{U}(\Lambda)$. Both thresholds are strictly declining in $\Lambda$.

\section{Proof Appendix.}

According to the optimal program, parents of both professions are willing to spend up to a constant fraction $C(\Lambda)$ of their income on their offspring's training. They educate their children if, and only if, the educational costs $h_{t} V_{t+1}$ do not exceed $C(\Lambda) y_{t}$. This translates into the threshold cost levels $\widetilde{h}^{S}(\Lambda)=C(\Lambda) m / \Lambda$ and $\widetilde{h}^{U}(\Lambda)=C(\Lambda) / \Lambda$. These critical costs being declining in $\Lambda$ has the following intuition. On the one hand, there is a direct effect of $\Lambda$ on the threshold values. For given $C(\Lambda)$, higher $\Lambda$ makes investments more expensive. Hence, a given education spending leads to lower threshold costs. On the other hand, $C(\Lambda)$ is decreasing in $\Lambda$, i.e. people's willingness to spend on education depends negatively on the growth rate as well. Parents are compensated for the education costs by higher income for their children, but also by a higher probability that later generations will be able to educate their children. The difference in probability to become educated between skilled and unskilled families is given by $\widetilde{h}^{S}-\widetilde{h}^{U}=C(m-1) / \Lambda$. For given $C$, increasing $\Lambda$ lowers this difference and thus educational gains. This diminishes the incentives to invest in education: agents are willing to expend a smaller fraction $C$ of their income on their offspring's training.

For the above investment behavior, the share of skilled workers develops according to $n_{t+1}=\widetilde{h}^{U}(\Lambda)\left[1+(m-1) n_{t}\right]$ and converges monotonically to

$$
n^{*}(\Lambda)=\frac{\widetilde{h}^{U}(\Lambda)}{1-\widetilde{h}^{U}(\Lambda)(m-1)} .
$$

Such an equilibrium is feasible if, and only if, the resulting $n$ sequence reinforces the growth rate $\Lambda: n^{*}(\underline{\Lambda}) \leq \tilde{n}$ or $n^{*}(\bar{\Lambda}) \geq \tilde{n}$. Conversely, such an equilibrium path does not exist if

$$
\widetilde{h}^{U}(\underline{\Lambda})[1+(m-1) \tilde{n}]>\tilde{n}>\widetilde{h}^{U}(\bar{\Lambda})[1+(m-1) \tilde{n}] .
$$

Because Proposition 2 shows that $\widetilde{h}^{U}(\bar{\Lambda})<\widetilde{h}^{U}(\underline{\Lambda})$, this condition will be fulfille for some parameter constellations. Thus,

Proposition 3 If (8) holds, growth rates fluctuate in any equilibrium. 


\section{Concluding remarks}

This paper shows that one need not refer to exogenous shocks to account for a nonmonotone pattern of growth and schooling activities. We fin that, in the presence of capital market imperfections, the interaction of skill acquisition and technological progress can generate endogenous growth in cycles. One might object that individual human capital investments exert an externality on economic growth so that some kind of learning-by-doing is the driving force behind technological improvements. This is the easiest way to formalize the link from human capital to growth. Similar results would likely obtain if there was a complementarity between research and development activities and education investments. ${ }^{15}$

Our model builds on an overlapping generations structure. While this suggests that cycles have a periodicity of at least two decades, similar interactions between productivity growth and human capital investment may also help explain cycles of lower periodicity. After an innovation, one has to decide whether or not to acquire the qualificatio necessary to work with the new method. Upgrading skills requires a time input and possibly pecuniary resources. ${ }^{16}$ If qualificatio requirements rise as the equipment becomes more productive, innovations or inventions make it more expensive for agents to invest in the most productive technologies. So the pattern of innovation has an impact on skill acquisition in a way which resembles the effects of our model. If one accepts this reasoning and that human capital stimulates productivity growth, cycles may occur in a model of infinitel lived agents. As mentioned above, one may even interpret our model with dynastic preferences in section 4 in this way. Since the lack of educational loans is crucial for the mechanism which we describe, it would be interesting to explore efficien y properties of various policy interventions in the field Owing to the positive character which our analysis has, we refrain from such an extension and leave it for future research.

\section{Appendix}

Proof of Lemma 1 Implicit differentiation of $u(x-H(x), m)=u(x, 1)$ shows that $H^{\prime}(x)>0$ if $u_{1}^{\prime}(x-H(x), m)>u_{1}^{\prime}(x, 1)$. W.1.o.g. we can choose $u$ to be linear homogenous. Then

$$
u_{1}^{\prime}(x-H(x), m)=u_{1}^{\prime}\left(\frac{x-H(x)}{m}, 1\right)>u_{1}^{\prime}(x, 1) .
$$

Here, the equality follows from linear homogeneity, and the inequality follows from the fact that a linear homogenous and strictly quasi-concave function is strictly concave in its arguments.

Proof of Proposition 1 Since the firs part is clear, it is sufficien to prove the stability result. Observe firs that the attracting set of the dynamic system (4) is the interval $I \equiv\left[\Phi_{2}(\tilde{n}), \Phi_{1}(\tilde{n})\right]$. We firs prove that $\Phi$ is injective on this set: any $n \in I$ has at most one preimage in $I$. $\Phi$ is injective on $I$ if $\Phi_{1}\left(\Phi_{2}(\tilde{n})\right)>\Phi_{2}\left(\Phi_{1}(\tilde{n})\right)$.

\footnotetext{
15 See Aghion and Howitt (1998) and the references therein.

16 Empirical evidence shows that the costs of productivity growth are rather costs of adopting existing technologies than costs of inventing new ones (see Jovanovic 1997).
} 
This condition is the same as $a_{1}\left(1-b_{2}\right)>a_{2}\left(1-b_{1}\right)$. Using the definitio of $a_{i}$ and $b_{i}$, this is the same as

$$
H\left(\frac{1}{\Lambda}\right)\left(1-H\left(\frac{m}{\bar{\Lambda}}\right)\right)>H\left(\frac{1}{\bar{\Lambda}}\right)\left(1-H\left(\frac{m}{\underline{\Lambda}}\right)\right)
$$

This inequality is fulfille because of Lemma 1 which proves that $\Phi$ is injective on $I$.

Any discontinuity of the $\tau$ th iterate $\Phi^{\tau}$ must be either $\tilde{n}$ or a preimage of $\tilde{n}$ of some iterate $\Phi^{s}, s=1, \ldots, \tau-1$. Since $\Phi$ and so all iterates $\Phi^{s}$ are injective on $I$, there can be at most $\tau$ discontinuities of $\Phi^{\tau}$ in $I$. This implies that there is a partition of $I$ into intervals $I_{s}, s=1, \ldots, m, m \leq \tau+1$, such that $\Phi^{\tau}$ is affine-linea on each $I_{s}$. Since the two maps $\Phi_{1}$ and $\Phi_{2}$ definin $\Phi$ are of the form $\Phi_{i}(n)=a_{i}+b_{i} n$ with $a_{i}>0$ and $b_{i}>0, \Phi^{\tau}$ can be written as $\Phi^{\tau}(n)=A_{s}+B_{s} n$ for $n \in I_{s}$ with positive constants $A_{s}$ and $B_{s}, s=1, \ldots, m$. Consider now a cycle $\left(n_{k}\right)_{k=1}^{\tau}$ of period $\tau$. Because this cycle is a fi ed point of $\Phi^{\tau}$, each $n_{k}$ must be contained in one of the intervals $I_{s}$ and no two elements can be contained in the same interval. This shows that $m \geq \tau$. Assume w.l.o.g. that $n_{k} \in I_{k}, k=1, \ldots, \tau$. From $n_{k}=A_{k}+B_{k} n_{k}$ and $A_{k}, n_{k}>0$, it follows $B_{k}<1$. Therefore, any trajectory with initial condition $n_{0} \in \cup_{s=1}^{\tau} I_{s}$ converges to the $\tau$-cycle. If $m=\tau+1, \Phi^{\tau}$ cannot have a fi ed point in $I_{m}$ (otherwise there would exist another cycle which must also enter the sets $I_{s}, s<m$, which is impossible). Hence, either $\Phi^{\tau}(n)>n$ for all $n \in I_{m}$ or $\Phi^{\tau}(n)<n$ for all $n \in I_{m}$. In any case, the trajectory leaves $I_{m}$ in finit time, enters $\cup_{s=1}^{\tau} I_{s}$ and converges to the $\tau$-cycle. This shows that the cycle is globally stable.

Proof of Proposition 2 Let $\bar{U}$ and $\underline{U}$ denote the expected life-time utility of a skilled and an unskilled household before the ability of the child is realized. Take $\bar{U}$ and $\underline{U}$ as given for a moment. A parent whose offspring is characterized by $h$ chooses education if the gains in expected utility compensate for forgone current consumption. Thus,

$$
\ln [z-\Lambda h]+\beta \bar{U} \geq \ln (z)+\beta \underline{U},
$$

must hold where $z$ equals 1 for unskilled and $m$ for skilled parents. There exist $\widetilde{h}^{S}$ and $\widetilde{h}^{U}$ in the interior of $[0,1]$ such that a skilled household buys education if $h \leq \widetilde{h}^{S}$ and an unskilled household if $h \leq \widetilde{h}^{U}$. These threshold cost levels $\widetilde{h}^{S}$ and $\widetilde{h}^{U}$ are given by

$$
-\ln [1-\Lambda h / z]=\beta[\bar{U}-\underline{U}]
$$

and satisfy $\widetilde{h}^{S}=m \widetilde{h}^{U}$.

To solve the maximization problem we must determine $\widetilde{h}^{S}, \widetilde{h}^{U}$, and $\bar{U}-\underline{U}$ in a consistent way: on the one hand, $\widetilde{h}^{S}$ and $\widetilde{h}^{U}$ depend on the difference in expected utilities via (9). On the other other hand, $\widetilde{h}^{S}$ and $\widetilde{h}^{U}$ must imply this very same difference when expected utilities are computed for these threshold values. ${ }^{17}$

${ }^{17}$ It is straightforward to show that a non-stationary solution is infeasible. 
Expected utilities $\underline{U}$ and $\bar{U}$ following for given $\widetilde{h}^{S}$ and $\widetilde{h}^{U}$ amount to:

$$
\bar{U}=\int_{0}^{\widetilde{h}^{S}} \ln [m-\Lambda h] d h+\beta \widetilde{h}^{S} \bar{U}+\int_{\widetilde{h}^{S}}^{1} \ln (m) d h+\beta\left(1-\widetilde{h}^{S}\right) \underline{U}
$$

and

$$
\underline{U}=\int_{0}^{\widetilde{h}^{U}} \ln [1-\Lambda h] d h+\beta \widetilde{h}^{U} \bar{U}+\beta\left(1-\widetilde{h}^{U}\right) \underline{U} .
$$

Some simplification yield

$$
\begin{aligned}
& \bar{U}=\ln m-\frac{m}{\Lambda}\left(1-\frac{\Lambda}{m} \widetilde{h}^{S}\right) \ln \left[1-\frac{\Lambda}{m} \widetilde{h}^{S}\right]-\widetilde{h}^{S}+\beta \underline{U}+\beta \widetilde{h}^{S}(\bar{U}-\underline{U}) \\
& \underline{U}=-\frac{1}{\Lambda}\left[1-\Lambda \widetilde{h}^{U}\right] \ln \left[1-\Lambda \widetilde{h}^{U}\right]-\widetilde{h}^{U}+\beta \underline{U}+\beta \widetilde{h}^{U}(\bar{U}-\underline{U}) .
\end{aligned}
$$

Making use of $\widetilde{h}^{S}=m \widetilde{h}^{U}$ and inserting for $\beta(\bar{U}-\underline{U})$ from (9) allows us to present these equations in a more convenient way:

$$
\begin{gathered}
\bar{U}=\ln m-\frac{m}{\Lambda} \ln \left[1-\frac{\Lambda}{m} \widetilde{h}^{S}\right]-\widetilde{h}^{S}+\beta \underline{U} \\
\underline{U}=-\frac{1}{\Lambda} \ln \left[1-\frac{\Lambda}{m} \widetilde{h}^{S}\right]-\frac{\widetilde{h}^{S}}{m}+\beta \underline{U} .
\end{gathered}
$$

Subtracting (11) from (10) implies the following difference between $\bar{U}$ and $\underline{U}$

$$
\bar{U}-\underline{U}=\ln m-\frac{m-1}{\Lambda}\left\{\ln \left[1-\frac{\Lambda}{m} \widetilde{h}^{S}\right]+\frac{\Lambda}{m} \widetilde{h}^{S}\right\}
$$

Note that $\Lambda \widetilde{h}^{S} / m$ is the fraction of income which households are willing to expend on their offspring's education. We denote this fraction $\Lambda \widetilde{h}^{S} / m$ by $C$ and obtain the following two different characterizations of $\bar{U}-\underline{U}$ from (9) and (12): $\bar{U}-\underline{U}=$ $-\ln [1-C] / \beta$ and $\bar{U}-\underline{U}=\ln m-(m-1)[\ln (1-C)+C] / \Lambda$. Hence, $C$ must solve:

$$
-\ln [1-C]\left[\frac{1}{\beta}-\frac{m-1}{\Lambda}\right]=\ln m-\frac{(m-1) C}{\Lambda} .
$$

Our assumption $m<\underline{\Lambda}$ implies that the LHS of (13) is strictly increasing in $C$. The RHS being decreasing in $C$, as well as LHS $(0)<\operatorname{RHS}(0)$ and LHS(1) $>$ RHS(1) guarantee the existence of a unique solution. Implicitly differentiating (13) shows that the solution depends negatively on $\Lambda$. Moreover, $\widetilde{h}^{S}(\bar{\Lambda})=C(\bar{\Lambda}) m / \bar{\Lambda}<$ $\widetilde{h}^{S}(\underline{\Lambda})=C(\underline{\Lambda}) / \underline{\Lambda}$. 


\section{References}

Aghion, P., Howitt, P.: Endogenous growth theory. Cambridge: MIT Press 1998

Azariadis, C.: The economics of poverty traps part one: complete markets. J Econ Growth 1, 449-486 (1996)

Azariadis, C., Drazen A.: Threshold externalities in economic development. Q J Econ 105, 501$526(1990)$

Bartel, A.P., Lichtenberg, F.R.: The comparative advantage of educated workers in implementing new technologies. Rev Econ Stat 69, 1-11 (1987)

Benabou, R.: Inequality and growth. NBER Macroeconomics Annual pp. 11-74. Cambridge: MIT Press 1996

Böhm, V., Kaas, L.: Differential savings, factor shares, and endogenous growth cycles. J Econ Dyn Control 24, 965-980 (2000)

Boldrin, M., Levine, D.K.: Growth cycles and market crashes. J Econ Theory 100, 13-39 (2001)

Caselli, F.: Technological revolutions. Am Econ Rev 89, 78-102 (1999)

Chiu, H.W.: Income inequality, human capital accumulation and economic performance. Econ J 108, 44-59 (1998)

De Gregorio, J., Kim, S.: Credit markets with differences in abilities: education, distribution, and growth. Int Econ Rev 41, 579-607 (2000)

DeJong, D.N., Ingram, B.F.: The cyclical behavior of skill acquisition. Rev Econ Dyn 4, 536-561 (2001)

Dellas, H., Sakellaris, P.: On the cyclicality of schooling: theory and evidence. Oxford Econ Pap 55, 148-172 (2003)

Erikson, R., Goldthorpe, J.: The constant flux a study of class mobility in industrial societies. Oxford: Clarendon Press 1992

Evans, G.W., Honkapohja, S. and Romer, P.M.: Growth cycles. Am Econ Rev 88, 495-515 (1998)

Jovanovic, B.: Learning and growth, advances in economics and econometrics: theory and applications. 7th World Congress, vol. 2. pp. 318-339 (1997)

Matsuyama, K.: Growing through cycles. Econometrica 67, 335-347 (1999)

Matsuyama, K.: Growing through cycles in an infinitel lived agent economy. J Econ Theo 100, 220-234 (2001)

Nelson, R.R., Phelps, E.S.: Investment in humans, technological diffusion, and economic growth. Am Econ Rev 56, 69-75 (1966)

Owen, A.L., Weil, D.N.: Intergenerational earnings mobility, inequality and growth. J Monet Econ 41, 71-104 (1998)

Piketty, T.: The dynamics of the wealth distribution and the interest rate with credit rationing. Rev Econ Stud 64, 173-189 (1997)

Schultz, T.W.: The value of ability to deal with disequilibria. J Econ Lit 13, 827-846 (1975)

Wälde, K.: The economic determinants of technology shocks in a real business cycle model. J Econ Dyn Control 27, 1-28 (2002) 